\title{
Granulocyte colony-stimulating factor in repeated IVF failure, a randomized trial
}

\author{
Ashraf Aleyasin ${ }^{1}$, Zhila Abediasl ${ }^{2}$, Atefeh Nazari ${ }^{3}$ and Mahdi Sheikh ${ }^{1,4}$ \\ ${ }^{1}$ Department of Infertility, Shariati Hospital, Tehran University of Medical Sciences, Tehran, Iran, ${ }^{2}$ Department of \\ Infertility, Bahman Hospital, Tehran, Iran, ${ }^{3}$ Omid Infertility Clinic, Tehran, Iran, and ${ }^{4}$ Maternal, Fetal and Neonatal \\ Research Center, Tehran University of Medical Sciences, Tehran, Iran
}

Correspondence should be addressed to M Sheikh; Email: mahdisheikh@gmail.com

\begin{abstract}
Recent studies have revealed key roles for granulocyte colony-stimulating factor (GCSF) in embryo implantation process and maintenance of pregnancy, and some studies showed promising results by using local intrauterine infusion of GCSF in patients undergoing in vitro fertilization (IVF). This multicenter, randomized, controlled trial included 112 infertile women with repeated IVF failure to evaluate the efficacy of systemic single-dose subcutaneous GCSF administration on IVF success in these women. In this study, the Long Protocol of ovarian stimulation was used for all participants. Sealed, numbered envelopes assigned 56 patients to receive subcutaneous $300 \mu \mathrm{g}$ GCSF before implantation and 56 in the control group. The implantation (number of gestational sacs on the total number of transferred embryos), chemical pregnancy (positive serum $\beta$-HCG), and clinical pregnancy (gestational sac and fetal heart) rates were compared between the two groups. This trial is registered at www.irct.ir (IRCT201503119568N11). The successful implantation $(18 \%$ vs $7.2 \%, P=0.007)$, chemical pregnancy $(44.6 \%$ vs $19.6 \%, P=0.005)$, and clinical pregnancy $(37.5 \%$ vs $14.3 \%, P=0.005$ ) rates were significantly higher in the intervention group than in the control group. After adjustment for participants' age, endometrial thickness, good-quality oocyte counts, number of transferred embryos, and anti-Mullerian hormone levels, GCSF treatment remained significantly associated with successful implantation $(\mathrm{OR}=2.63,95 \% \mathrm{CI}=1.09-6.96)$, having chemical pregnancy $(\mathrm{OR}=2.74,95 \% \mathrm{Cl}=1.11-7.38)$ and clinical pregnancy $(\mathrm{OR}=2.94,95 \% \mathrm{Cl}=1.23-8.33)$. In conclusion, administration of single-dose systemic subcutaneous GCSF before implantation significantly increases the IVF success, implantation, and pregnancy rates in infertile women with repeated IVF failure.

Reproduction (2016) 151 637-642
\end{abstract}

\section{Introduction}

In vitro fertilization (IVF) is the most successful assisted reproductive technology (ART) used to treat infertility. However, IVF is expensive and time-consuming, and the success rate is still less than $40 \%$. Many couples still remain unsuccessful after several IVF attempts causing deep effect on quality of life and a heavy financial burden (Zeyneloglu \& Onalan 2014). With the recent advances in understanding the important role of granulocyte colony-stimulating factor (GCSF) in reproduction, and the detection of GCSF receptors in placental cytotrophoblasts, syncytiotrophoblasts, decidual stromal cells, endometrial gland cells, and follicular cells (Saito et al. 1994, Miyama et al. 1998, McCracken et al. 1999, Salmassi et al. 2005, Ledee et al. 2008, 2013, Rahmati et al. 2014), GCSF started to attract attentions in gynecology, especially in patients with recurrent abortions and/or infertility.

In 2009, GCSF was successfully used in patients with recurrent abortions (Scarpellini \& Sbracia 2009), and later another study reported promising results by using GCSF in four women undergoing IVF who had demonstrated highly inadequate endometrium (Gleicher et al. 2011). Since then, several studies with controversial results have evaluated the effect of GCSF on endometrial thickness and IVF success (Scarpellini \& Sbracia 2012, Gleicher et al. 2013, Barad et al. 2014, Eftekhar et al. 2014, Kunicki et al. 2014, Li et al. 2014, $\mathrm{Xu}$ et al. 2015). In the studies of Scarpellini and coworkers and $\mathrm{Xu}$ and coworkers, the implantation and pregnancy rates were significantly higher in IVF patients treated with GCSF (Scarpellini \& Sbracia 2012, Xu et al. 2015), whereas in the studies of Barad and coworkers and $\mathrm{Li}$ and coworkers, treatment with GCSF did not affect endometrial thickness, implantation, or pregnancy rates in IVF patients (Barad et al. 2014, Li et al. 2014).

These studies had some limitations that might preclude the researchers from making a precise conclusion, including small sample size (Gleicher et al. 2013, Eftekhar et al. 2014, Kunicki et al. 2014, Li et al. 2014, Xu et al. 2015), no control groups (Gleicher et al. 2013, Kunicki et al. 2014), no 
randomization (Gleicher et al. 2013, Eftekhar et al. 2014, Kunicki et al. 2014, Li et al. 2014), and unintended selection bias (Scarpellini \& Sbracia 2012, Barad et al. 2014, Li et al. 2014, Xu et al. 2015). All these studies have suggested that large, randomized, controlled trials are still needed to evaluate the efficacy of GCSF administration on IVF success (Scarpellini \& Sbracia 2012, Gleicher et al. 2013, Barad et al. 2014, Eftekhar et al. 2014, Kunicki et al. 2014, Li et al. 2014, Xu et al. 2015). The efficacy of GCSF in patients with repeated IVF failure has not been established yet. In addition, most studies have infused GCSF locally into the uterine cavity. Some authors have suggested that systemic administration of GCSF might be more effective than local infusion (Barad et al. 2014, Zeyneloglu et al. 2013).

We conducted this study on infertile women with repeated IVF failure to evaluate the efficacy of systemic subcutaneous GCSF administration on IVF success, implantation, and pregnancy rates.

\section{Materials and methods \\ Study population and design}

This multicenter, prospective, randomized, open-label, controlled trial was conducted on 112 infertile women who had repeated IVF failure. The study was conducted at the infertility departments of Shariati Hospital (46 patients), Bahman Hospital (36 patients), and Omid Infertility Clinic (30 patients), Tehran, Iran, from March 2015 through January 2016.

Repeated IVF failure is determined when transferred embryos fail to implant after several IVF cycles. However, there are no formal criteria defining the number of failed cycles or the total number of embryos transferred in these IVF attempts (Simon \& Laufer 2012). In our study, repeated IVF failure was defined as failure of implantation in at least three consecutive IVF attempts, in which three embryos of high-grade quality are transferred in each cycle (Simon \& Laufer 2012).

The patients were considered eligible if they had the following inclusion criteria: age $<40$ years, having repeated IVF failure, absence of contraindications for GCSF treatment (sickle cell disease, chronic neutropenia, known past or present malignancy, renal insufficiency, upper respiratory infection, pneumonia, and congenital fructose intolerance), and absence of any systemic disease (including hypertension, diabetes, antiphospholipid syndrome, systemic lupus erythematosus, thrombophilia, and thyroid disorders). The exclusion criteria were the presence of an inadequate endometrium $(<7 \mathrm{~mm})$ on the day of hCG injection; the detection of Asherman's syndrome, fibroids, and/or polyps via diagnostic hysteroscopy; and/or refusing to give an informed consent to participate in the study.

A computerized random number generator was used for sequence generation. Simple randomization with a 1:1 allocation ratio was used. Consecutive opaque envelopes for the allocation concealment were performed. The envelopes were opaque when held to the light, opened sequentially, and only after the participant's name and other details were written on the appropriate envelope and implementation of assignments was carried out.

\section{Intervention, data, and specimen collection}

In this study, the Long Protocol of ovarian stimulation was used for all the participants. Gonadotropin-releasing hormone $(\mathrm{GnRH})$ agonist $0.1 \mathrm{mg}$ (Decapeptyl; Ferring) was administered

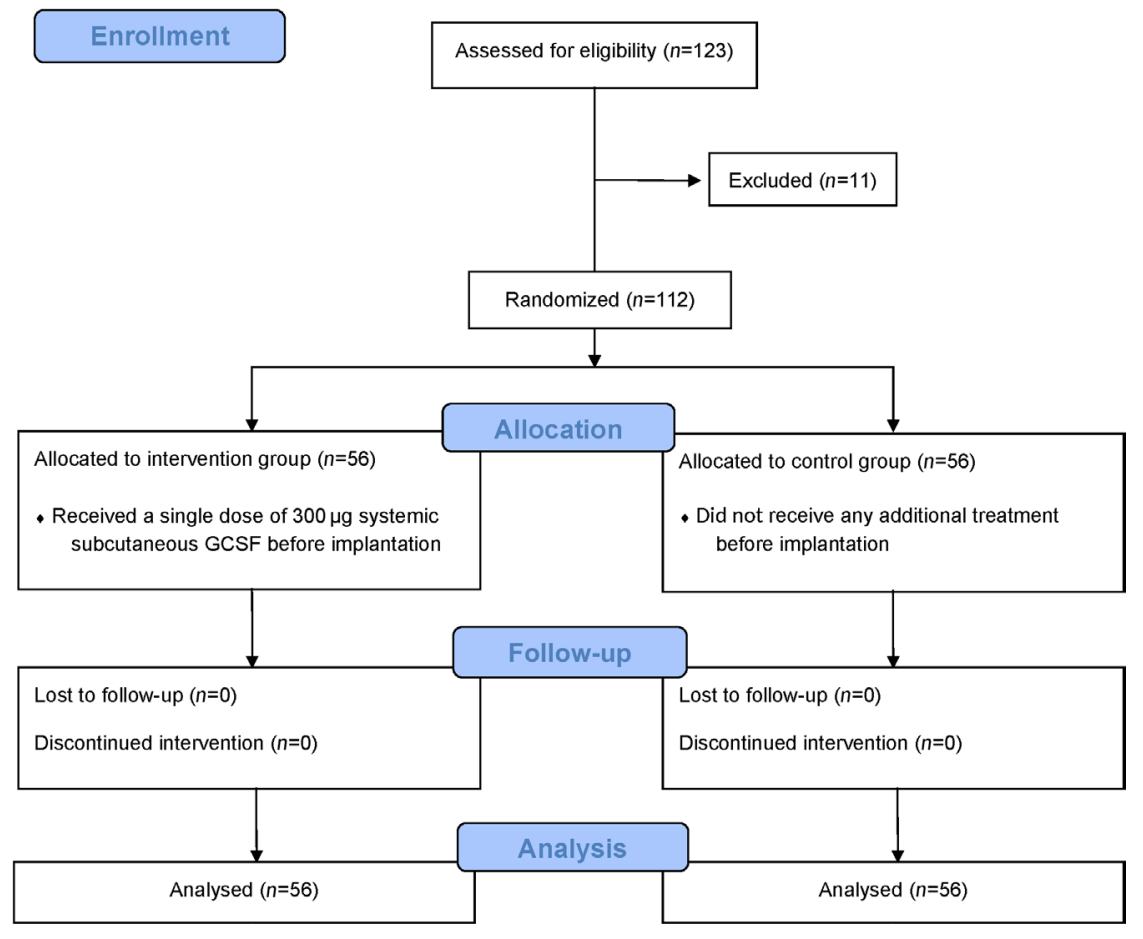

Reproduction (2016) 151 637-642
Figure 1 CONSORT flow diagram of the study. 
subcutaneously once/daily, which was started 7 days before the next expected period and continued until the third day of the cycle. Next, a daily subcutaneous injection of 300 IU folliclestimulating hormone (FSH) (Gonal-F; Merck Serono) was applied for 4 days starting from the third day of the cycle. On the 6th day of the cycle, 150-300 IU intramuscular injection of human menopausal gonadotropin (hMG) (Menogon; Ferring) was administered. The hMG dose was administered based on the participants' weight, FSH level, and antral follicle counts (AFC). Transvaginal sonography was performed every 1-3 days to evaluate the follicle size. When at least two follicles reached a minimum of $18 \mathrm{~mm}$ diameter, 10,000 IU intramuscular injection of human chorionic gonadotropin (hCG) (Choragon; Ferring) was given to the participants. All the participants received $5 \mathrm{mg}$ prednisolone daily starting from the day of hCG injection until 3 days following oocyte retrieval. Transvaginal oocyte pickup was performed $36 \mathrm{~h}$ after hCG administration under transvaginal sonography. Embryo quality was evaluated using standard day-3 embryo grading; day-3 embryos with eight cells and $<15 \%$ fragmentation were considered good. About one to three good embryos were transferred into the uterine cavity. The number of transferred embryos was chosen based on the criteria of the American Society for Reproductive Medicine (ASRM) (Practice Committee of the American Society for Reproductive Medicine 2013). The participants were randomly allocated to two groups: the intervention (GCSF) group and the control group. In the intervention group, a single dose of $300 \mu \mathrm{g}$ GCSF (Neupogen; Roche) was administered subcutaneously $1 \mathrm{~h}$ before the embryo transfer. The participants in the control group did not receive any additional treatment before the embryo transfer. Luteal phase support was carried out on all participants using Cyclogest suppositories two times per day vaginally and $80 \mathrm{mg}$ aspirin daily. After 2 weeks of the embryo transfer, serum $\beta$-HCG level was measured. After 4 and 6 weeks of embryo transfer, transvaginal sonography was performed on the participants who had a positive pregnancy test to detect the gestational sac and fetal heart rate (FHR) respectively.

The outcomes were chemical pregnancy, clinical pregnancy, and implantation rates. Chemical pregnancy was defined as having a positive serum $\beta$-HCG test, whereas clinical pregnancy was defined as the presence of gestational sac and fetal heart on ultrasound examination, and implantation rates were determined by the number of gestational sacs at least 28 days after embryo transfer based on the total number of embryos transferred per group.

This study was approved by the Research Deputy and the Ethics Committee of the Tehran University of Medical Sciences. This study was registered prospectively at the Iranian Registry of Clinical Trials (www.irct.ir), which is a Primary Registry in the WHO Registry Network (Registration Number $=$ IRCT201503119568N11).

\section{Statistical analysis}

Presuming a clinical pregnancy rate of $15 \%$ in the control group and anticipating a clinical pregnancy rate of $40 \%$ with GCSF treatment based on the available literature, at least 50 participants in each study arm would be needed for $80 \%$ power, $\beta=20 \%$ and $\alpha=0.05$. All the statistical analyses were performed using SPSS statistical software (version 18.0.0; PASW, Chicago, IL, USA). Data were expressed as mean, S.D., and percentage. Mean comparisons between the two groups and variables were performed using the $t$-test for independent samples and $t$-test for paired samples depending on the tested variables. Furthermore, the Pearson correlation coefficient, $\chi^{2}$ analysis, Fisher's exact test, and one-way ANOVA were also used. The level of statistical significance was set at $P$ value $<0.05$.

\section{Results \\ Descriptive statistics}

In total, 123 infertile women with repeated IVF failure agreed to participate in the study, 12 of whom were excluded. Of those excluded, six had inadequate endometrium, three were $>40$ years of age, and two had $<3$ failed IVF attempts. Therefore, 112 infertile women with $\geq 3$ failed IVF attempts remained in the study. Of the 112 women, 56 were randomly assigned to the intervention group to receive a single dose of subcutaneous GCSF on the embryo transfer day and 56 were allocated to the control group (Fig. 1). Upon enrollment, the mean \pm s.D. for the participants' age was $33 \pm 4.8$ years; their partners' age was $38.5 \pm 8.2$ years; the participants' weight was $67.9 \pm 10.1 \mathrm{~kg}$; the infertility duration was $8.1 \pm 5$ years; anti-Mullerian hormone $(\mathrm{AMH})$ level was $2.8 \pm 2.5$ (median: 2.45) $\mathrm{ng} / \mathrm{mL}$; $\mathrm{FSH}$ level was $7.3 \pm 4.1 \mathrm{mIU} / \mathrm{mL}$; luteinizing hormone $(\mathrm{LH})$ level was $8 \pm 5.1 \mathrm{mIU} / \mathrm{mL}$; antral follicular counts (AFC) were $10.7 \pm 4.9$ (median: 10); endometrial thickness on the day of embryo transfer was $11.3 \pm 2.1 \mathrm{~mm}$; retrieved

Table 1 Comparison of the demographics between the intervention and the control groups. Data are presented as mean \pm S.D.

\begin{tabular}{lccc}
\hline Demographics & $\begin{array}{c}\text { GCSF-treated } \\
\text { group } n=56\end{array}$ & $\begin{array}{c}\text { Control } \\
\text { group } n=56\end{array}$ & $\boldsymbol{P}$ value \\
\hline Participants' age (years) & $33.5 \pm 4.2$ & $32.4 \pm 5.2$ & 0.22 \\
The partners' age (years) & $40.1 \pm 9.5$ & $37 \pm 6.4$ & 0.08 \\
Weight (kg) & $66.7 \pm 9.2$ & $69.1 \pm 10.9$ & 0.19 \\
Infertility duration (years) & $8.1 \pm 4.2$ & $8.2 \pm 5.8$ & 0.89 \\
AMH (ng/mL; median) & 2.05 & 2.55 & 0.06 \\
FSH (mIU/mL) & $7.5 \pm 4.6$ & $7.2 \pm 3.6$ & 0.65 \\
LH (mIU/mL) & $8.9 \pm 5.9$ & $7.2 \pm 4.2$ & 0.09 \\
AFC (median) & 10 & 10 & 0.11 \\
Endometrial thickness (mm) & $11.2 \pm 2$ & $11.5 \pm 2.3$ & 0.9 \\
Retrieved oocyte counts & $15.8 \pm 7.2$ & $14.3 \pm 6.5$ & 0.1 \\
Good-quality MII oocyte counts & $9.1 \pm 5.1$ & $8.2 \pm 6.1$ & 0.07 \\
Transferred embryos (number) & $2.3 \pm 0.6$ & $2.5 \pm 0.6$ & 0.19 \\
Failed IVF attempts & 3 & 3 & 0.62 \\
(number; median) & & & \\
Primary infertility (number (\%)) & $51(91.1 \%)$ & $47(83.9 \%)$ & 0.25 \\
\hline
\end{tabular}

GCSF, granulocyte colony-stimulating factor; S.D., standard deviation; $\mathrm{AMH}$, anti-Mullerian hormone; $\mathrm{FSH}$, follicle-stimulating hormone; $\mathrm{LH}$, luteinizing hormone; AFC, antral follicular counts; MII, metaphase II; IVF, in vitro fertilization. 
Table 2 Comparison of the IVF outcomes between the intervention and the control groups.

\begin{tabular}{|c|c|c|c|c|}
\hline Outcomes & $\begin{array}{c}\text { GCSF group } \\
n=56\end{array}$ & $\begin{array}{c}\text { Control group } \\
n=56\end{array}$ & $\begin{array}{c}\text { Unadjusted OR } \\
(95 \% \mathrm{Cl})\end{array}$ & $\begin{array}{c}\text { Adjusted } \mathbf{O R}^{+} \\
(95 \% \mathrm{Cl})\end{array}$ \\
\hline Implantation (number of GS/total transferred embryos (\%)) & 24/133 (18\%) & 10/138 (7.2\%) & $2.81(1.29-6.15)$ & $2.63(1.09-6.96)$ \\
\hline Chemical pregnancy (number (\%)) & $25(44.6 \%)$ & $11(19.6 \%)$ & $3.29(1.41-7.67)$ & $2.74(1.11-7.38)$ \\
\hline Clinical pregnancy (number (\%)) & $21(37.5 \%)$ & $8(14.3 \%)$ & $3.6(1.43-9.06)$ & $2.94(1.23-8.33)$ \\
\hline Ectopic pregnancy (number $(\%)$ ) & $2(3.6 \%)$ & $1(1.8 \%)$ & $2.03(0.17-23.13)$ & - \\
\hline
\end{tabular}

GCSF, granulocyte colony-stimulating factor; OR, odds ratio; $95 \% \mathrm{Cl}, 95 \%$ confidence interval; GS, gestational sacs.

${ }^{\dagger}$ Adjusted for the participants' age, endometrial thickness, good-quality metaphase II oocyte counts, number of transferred embryos, and anti-Mullerian hormone levels.

oocyte counts were $14.9 \pm 7$; retrieved good-quality (metaphase II (MII)) oocyte counts was $8.5 \pm 5.6$; and the number of transferred embryos was $2.4 \pm 0.6$. Of the 112 participants, 98 had primary infertility $(87.5 \%)$, while 14 had secondary infertility $(12.5 \%)$. Of the 112 participants, 57 had three failed IVF attempts (50.8\%), 27 had four failed IVF attempts (24.1\%), and $28 \mathrm{had} \geq 5$ failed IVF attempts (25\%).

There were no statistically significant differences in the demographics of the patients allocated to the intervention group versus those allocated to the control group (Table 1).

\section{The effect of GCSF on IVF outcomes in patients with repeated IVF failure}

The implantation rate in the intervention group was significantly higher than that in the control group $(18 \%$ vs $7.2 \%, P=0.007)$. The chemical pregnancy rate was significantly higher in the intervention group than in the control group ( $44.6 \%$ vs $19.6 \%, P=0.005)$. The clinical pregnancy rate was also significantly higher in the intervention group than in the control group $37.5 \%$ vs $14.3 \%, P=0.005)$. One participant in each group had ectopic pregnancy $(3.6 \%$ vs $1.8 \%, P=1)$ (Table 2$)$.

\section{Dependency of the obtained results}

Logistic regression model was used to determine the dependency of the effect of GCSF treatment on IVF outcomes. When adjusted for the participants' age, endometrial thickness, good-quality MII oocyte counts, number of transferred embryos, and AMH levels, GCSF treatment remained significantly associated with successful implantation $(\mathrm{OR}=2.63,95 \% \mathrm{Cl}=1.09$ 6.96), having chemical pregnancy $(\mathrm{OR}=2.74,95 \%$ $\mathrm{Cl}=1.11-7.38)$, and having clinical pregnancy $(\mathrm{OR}=2.94,95 \% \mathrm{Cl}=1.23-8.33)$ (Table 2).

\section{Discussion}

\section{GCSF and its use in obstetrics}

GCSF is a glycoprotein with growth factor and cytokine functions that is produced in different tissues. GCSF was first cloned and used in the late 1980s and since then is being used mainly in patients with bone marrow suppression and severe neutropenia (Morstyn et al. 1988). In 1989, Uzumaki and coworkers identified the GCSF receptors in human placental membranes and trophoblastic cells and suggested that GCSF might play some role in the fetoplacental unit during human development (Uzumaki et al. 1989). After that, studies began to detect GCSF receptors in different parts of the female reproductive system and at the feto-placental interface; GCSF receptors were identified in endometrial gland cells, follicular cells, placental cytotrophoblasts and syncytiotrophoblasts, decidual stromal cells, and fetal membranes (Saito et al. 1994, Miyama et al. 1998, McCracken et al. 1999, Ledee et al. 2008). These advances, in addition to the reports of the safety of GCSF use in pregnancy (Dale et al. 2003), made the researchers think of using GCSF in obstetrics. In 2009, Scarpellini \& Sbracia for the first time used GCSF in 86 women with unexplained recurrent abortion and showed that $82 \%$ of the GCSF-treated women delivered a healthy neonate compared with $48 \%$ of the controls; additionally, in their study, GCSF was completely safe and was not associated with any adverse maternal or neonatal outcomes (Scarpellini \& Sbracia 2009).

\section{The effect of GCSF on implantation and pregnancy in IVF}

In our study, subcutaneous administration of GCSF independently improved the successful implantation and pregnancy rates in IVF patients with normal endometrium who had repeated implantation failure after several IVF attempts. This was in accordance with the study of Scarpellini \& Sbracia in women with repeated IVF failure that documented higher implantation rate in IVF women who had received GCSF compared with the placebo group (Scarpellini \& Sbracia 2012). In contrast, in three other studies, GCSF administration did not affect the implantation or pregnancy rates (Barad et al. 2014, Eftekhar et al. 2014, Li et al. 2014). The differences in administration route, participants' age, endometrial thickness, and sample sizes could have contributed to the observed differences in the results; in the study of Scarpellini \& Sbracia and our study, the sample size was larger, GCSF was administered systemically through the subcutaneous route, the study populations were younger, and had normal proliferating 
endometrium (Scarpellini \& Sbracia 2012), whereas in other studies, GCSF was administered locally through intrauterine perfusion, the sample sizes were smaller, the endometrium was thin in two studies, and the participants were older in the third study (Barad et al. 2014, Eftekhar et al. 2014, Li et al. 2014).

\section{The role of GCSF in implantation and pregnancy}

Our results indicate that GCSF might play an important role in the implantation process and in the maintenance of pregnancy. Salmassi et al. evaluated 93 women who underwent IVF; in their study, patients who became pregnant revealed a continuous increase in serum GCSF level from the day of embryo transfer to the day of implantation and from the day of confirmation of pregnancy to gestation; in contrast, those patients who did not become pregnant initially showed a slight increase in GCSF level, then with the failure of implantation as the GCSF level decreased significantly (Salmassi et al. 2005). The authors concluded that this cytokine could have an important function in achieving or maintaining pregnancy (Salmassi et al. 2005). In another study, Rahmati and coworkers demonstrated significantly lower levels of GCSF receptors at the maternal-fetal interface in infertile women with implantation failure, and interestingly, stimulation with high-dose GCSF was able to increase the expression of GCSF receptors in these patients (Rahmati et al. 2014).

GCSF might affect reproduction, implantation, and pregnancy through several possible mechanisms; GCSF has been shown to induce the trophoblast proliferation, invasion, and maintenance during pregnancy (Miyama et al. 1998, McCracken et al. 1999). GCSF also plays a key role during the embryo implantation process; it modulates fundamental genes responsible for the local embryo adhesion, cell migration, tissue remodeling, and angiogenesis, which are unavoidable for a successful implantation and further placentation (Rahmati et al. 2014). Finally, GCSF might be involved in the induction of adaptive changes that favor the immune tolerance in pregnancy; due to the semi-allogenic nature of the fetus, pregnancy represents an immune challenge to the mother. GCSF switches the T-cell cytokine secretion profile to the Th2 responses and promotes the IL-10producing regulatory $T$ cells and tolerogenic dendritic cell differentiation (Rutella et al. 2005), which are important parts of the immunoregulatory events that occur before and after the implantation in the uterus (Ledee et al. 2008).

\section{The ideal route and dose of GCSF administration}

The ideal route and dose of GCSF administration in obstetrics have not been identified yet. It seems that systemic administration is more effective and associated with a better success rate than the commonly used local intrauterine infusion (Scarpellini \& Sbracia 2012, Zeyneloglu et al. 2013, Barad et al. 2014). In this study and the study of Scarpellini \& Sbracia GCSF was administered systemically through subcutaneous injection (Scarpellini \& Sbracia 2012). Scarpellini \& Sbracia used daily subcutaneous injection of $60 \mu \mathrm{g}$ GCSF from the day of embryo transfer to the day of $\beta$-HCG test, and if it was positive, the treatment was continued for another 40 days, while we used a singledose subcutaneous injection of $300 \mu \mathrm{g}$ GCSF only before the implantation. In both studies, the intervention group had significantly higher implantation and pregnancy rates than the control group, while it is obvious that the financial burden in our study is lower and that a singledose administration is much easier, safer, and more tolerable than daily administration for a long time.

\section{Strengths and limitations}

The main strengths of our study were its randomized, controlled design and to evaluate for the first time the efficacy of systemic subcutaneous GCSF administration on IVF success in women with repeated IVF failure. Assessing the administration of systemic and singledose GCSF, which is easier, more tolerable and more cost-effective than repeated administration or local infusion of GCSF were other strengths of this study. This study, however, had some limitations that should be considered when interpreting the results; due to the nature of the study, it was unblinded and no placebo was used that could potentially affect the results of the study. In addition, we did not evaluate the pregnancy outcomes in the two groups of the study; this could help us in better interpretation of the results and establishing the safety of GCSF administration.

In conclusion, systemic administration of singledose $300 \mu \mathrm{g}$ GCSF subcutaneously 1 hour before embryo transfer significantly increases the IVF success, implantation, and pregnancy rates in infertile women with repeated IVF failure, which is independent of the women's age, endometrial thickness, good-quality oocyte counts, number of transferred embryos, and $\mathrm{AMH}$ levels. Further randomized, controlled trials are required to confirm these results and to establish the best rout and dose of GCSF administration in IVF patients. Also studies are required to compare the efficacy, side effects, and pregnancy outcomes of the intrauterine perfusion versus systemic subcutaneous administration of GCSF in IVF patients.

\section{Declaration of interest}

The authors declare that there is no conflict of interest that could be perceived as prejudicing the impartiality of the research reported. 


\section{Funding}

This Research was funded by the Research Deputy of the Tehran University of Medical Sciences (Grant Number: 10010317).

\section{Acknowledgements}

The authors thank the personnel of infertility departments of Shariati and Bahman hospitals and Omid Infertility Clinic for their help in performing the study and data collection.

\section{References}

Barad DH, Yu Y, Kushnir VA, Shohat-Tal A, Lazzaroni E, Lee HJ \& Gleicher N 2014 A randomized clinical trial of endometrial perfusion with granulocyte colony-stimulating factor in in vitro fertilization cycles: impact on endometrial thickness and clinical pregnancy rates. Fertility and Sterility 101 710-715. (doi:10.1016/j.fertnstert.2013.12.016)

Dale DC, Cottle TE, Fier CJ, Bolyard AA, Bonilla MA, Boxer LA, Cham B, Freedman MH, Kannourakis G, Kinsey SE et al. 2003 Severe chronic neutropenia: treatment and follow-up of patients in the Severe Chronic Neutropenia International Registry. American Journal of Hematology 72 82-93. (doi:10.1002/(ISSN)1096-8652)

Eftekhar M, Sayadi M \& Arabjahvani F 2014 Transvaginal perfusion of G-CSF for infertile women with thin endometrium in frozen ET program: a non-randomized clinical trial. Iranian Journal of Reproductive Medicine 12 661-666.

Gleicher N, Vidali A \& Barad DH 2011 Successful treatment of unresponsive thin endometrium. Fertility and Sterility 95 2123.e132123.e17. (doi:10.1016/j.fertnstert.2011.01.143)

Gleicher N, Kim A, Michaeli T, Lee HJ, Shohat-Tal A, Lazzaroni E \& Barad DH 2013 A pilot cohort study of granulocyte colony-stimulating factor in the treatment of unresponsive thin endometrium resistant to standard therapies. Human Reproduction 28 172-177. (doi:10.1093/ humrep/des370)

Kunicki M, Lukaszuk K, Woclawek-Potocka I, Liss J, Kulwikowska P \& Szczyptanska J 2014 Evaluation of granulocyte colony-stimulating factor effects on treatment-resistant thin endometrium in women undergoing in vitro fertilization. BioMed Research International 2014913235. (doi:10.1155/2014/913235)

Ledee N, Lombroso R, Lombardelli L, Selva J, Dubanchet S, Chaouat G, Frankenne F, Foidart JM, Maggi E, Romagnani S et al. 2008 Cytokines and chemokines in follicular fluids and potential of the corresponding embryo: the role of granulocyte colony-stimulating factor. Human Reproduction 23 2001-2009. (doi:10.1093/humrep/den192)

Ledee N, Gridelet V, Ravet S, Jouan C, Gaspard O, Wenders F, Thonon F, Hincourt N, Dubois M, Foidart JM et al. 2013 Impact of follicular G-CSF quantification on subsequent embryo transfer decisions: a proof of concept study. Human Reproduction 28 406-413. (doi:10.1093/ humrep/des354)

Li Y, Pan P, Chen X, Li L \& Yang D 2014 Granulocyte colony-stimulating factor administration for infertile women with thin endometrium in frozen embryo transfer program. Reproductive Sciences 21 381-385. (doi:10.1177/1933719113497286)
McCracken SA, Grant KE, MacKenzie IZ, Redman CW \& Mardon HJ 1999 Gestational regulation of granulocyte-colony stimulating factor receptor expression in the human placenta. Biology of Reproduction 60 790-796. (doi:10.1095/biolreprod60.4.790)

Miyama M, Umesaki N \& Kawabata M 1998 Identification of the granulocyte colony-stimulating factor (G-CSF) producing cell population in human decidua and its biological action on trophoblast cell. Osaka City of Medical Journal 44 85-96.

Morstyn G, Campbell L, Souza LM, Alton NK, Keech J, Green M, Sheridan W, Metcalf D \& Fox R 1988 Effect of granulocyte colony stimulating factor on neutropenia induced by cytotoxic chemotherapy. Lancet 1 667-672.

Practice Committee of the American Society for Reproductive Medicine, Practice Committee of the Society for Assisted Reproductive Technology 2013 Criteria for number of embryos to transfer: a committee opinion. Fertility and Sterility 99 44-46. (doi:10.1016/j.fertnstert.2012.09.038)

Rahmati M, Petitbarat M, Dubanchet S, Bensussan A, Chaouat G \& Ledee N 2014 Granulocyte-colony stimulating factor related pathways tested on an endometrial ex-vivo model. PLOS ONE 9 e102286. (doi:10.1371/ journal.pone.0102286)

Rutella S, Zavala F, Danese S, Kared H \& Leone G 2005 Granulocyte colony-stimulating factor: a novel mediator of $\mathrm{T}$ cell tolerance. Journal of Immunology 175 7085-7091. (doi:10.4049/jimmunol.175.11.7085)

Saito S, Fukunaga R, Ichijo M \& Nagata S 1994 Expression of granulocyte colony-stimulating factor and its receptor at the fetomaternal interface in murine and human pregnancy. Growth Factors 10 135-143. (doi:10.3109/08977199409010987)

Salmassi A, Schmutzler AG, Schaefer S, Koch K, Hedderich J, Jonat W \& Mettler L 2005 Is granulocyte colony-stimulating factor level predictive for human IVF outcome? Human Reproduction 20 2434-2440. (doi:10.1093/humrep/dei071)

Scarpellini F \& Sbracia M 2009 Use of granulocyte colony-stimulating factor for the treatment of unexplained recurrent miscarriage: a randomised controlled trial. Human Reproduction 24 2703-2708. (doi:10.1093/humrep/dep240)

Scarpellini F \& Sbracia M 2012 G-CSF treatment improves IVF outcome in women with recurrent implantation failure in IVF. Journal of Reproductive Immunology 94 103. (doi:10.1016/j.jri.2012.03.435)

Simon A \& Laufer N 2012 Assessment and treatment of repeated implantation failure (RIF). Journal of Assisted Reproduction and Genetics 29 1227-1239. (doi:10.1007/s10815-012-9861-4)

Uzumaki H, Okabe T, Sasaki N, Hagiwara K, Takaku F, Tobita M, Yasukawa K, Ito S \& Umezawa Y 1989 Identification and characterization of receptors for granulocyte colony-stimulating factor on human placenta and trophoblastic cells. PNAS 86 9323-9326. (doi:10.1073/ pnas.86.23.9323)

Xu B, Zhang Q, Hao J, Xu D \& Li Y 2015 Two protocols to treat thin endometrium with granulocyte colony-stimulating factor during frozen embryo transfer cycles. Reproductive BioMedicine Online 30 349-358. (doi:10.1016/j.rbmo.2014.12.006)

Zeyneloglu HB \& Onalan G 2014 Remedies for recurrent implantation failure. Seminars in Reproductive Medicine 32 297-305. (doi:10.1055/ s-0034-1375182)

Zeyneloglu HB, Onalan G, Durak T, Alyazici I \& Unal E 2013 Granulocyte macrophage colony stimulating factor (G-CSF) administration for art patients with repeated implantation failure (RIF): which route is best? Fertility and Sterility $\mathbf{1 0 0}$ S291-S292. (doi:10.1016/ j.fertnstert.2013.07.1031)

Received 25 January 2016

First decision 7 March 2016

Revised manuscript received 9 March 2016

Accepted 15 March 2016 